\title{
EVALUACIÓN DEL DESEMPEÑO EN EL DIAGNÓSTICO MICROSCÓPICO DE MALARIA EN LA RED DE LABORATORIOS DEL INSTITUTO NACIONAL DE SALUD DE PERÚ, 2012-2017
}

\author{
Carlos A. Bartra-More ${ }^{1, a}$, José Ramos-Castillo2,3,b, Roberto C. Mendoza-Bautista1,a, \\ Nadia R. Galindo-Cabello1,c, Melitón Gebol-Cahuaza1,d, Franklin J. Chirinos-Palomino1,a
}

\begin{abstract}
RESUMEN
El diagnóstico oportuno de malaria es una estrategia propuesta por la Organización Mundial de la Salud para reducir la morbimortalidad por esta enfermedad. Se realizó un estudio con el objetivo de evaluar el desempeño en el diagnóstico microscópico de malaria en la red de laboratorios pertenecientes al Laboratorio de Referencia Nacional de Malaria del Instituto Nacional de Salud entre 2012 y 2017. En los años de estudio, los laboratorios tuvieron la calificación de aceptable en el diagnóstico de Plasmodium en un $38,4 \%, 43,7 \%, 60,0 \%, 83,3 \%, 90,9 \%$ y $95,8 \%$, respectivamente; en la evaluación de especie en un $0 \%, 6,2 \%, 15,0 \%, 50,0 \%, 40,9 \%$ y $54,1 \%$, respectivamente; en la evaluación de estadio en un $23,0 \%, 25,0 \%, 35,0 \%, 83,3 \%, 54,5 \%$ y $79,1 \%$, respectivamente; y en la evaluación de densidad parasitaria en un $0 \%, 6,2 \%, 10,0 \%, 33,3 \%, 0 \%$ y $12,5 \%$, respectivamente. Concluimos que en el periodo evaluado se incrementó el porcentaje de laboratorios que diagnosticaron, reconocieron la especie y estadio, mas no el reconocimiento de densidad parasitaria.
\end{abstract}

Palabras clave: Malaria; Plasmodium; Diagnóstico; Laboratorios; Perú (fuente: DeCS BIREME).

\section{PERFORMANCE ASSESSMENT IN MICROSCOPIC DIAGNOSIS OF MALARIA IN THE LABORATORY NETWORK OF THE NATIONAL INSTITUTE OF HEALTH OF PERU, 2012-2017}

\begin{abstract}
Timely diagnosis of malaria is a strategy proposed by the World Health Organization to reduce malaria morbidity and mortality. A study was conducted to assess performance in microscopic diagnosis of malaria in the network of laboratories under the National Malaria Reference Laboratory of the National Institute of Health between 2012 and 2017. In the years of study, the laboratories obtained a rating of "acceptable" in the diagnosis of Plasmodium by $38.4 \%, 43.7 \%, 60.0 \%$, $83.3 \%, 90.9 \%$, and $95.8 \%$, respectively, in the evaluation of species by $0 \%, 6.2 \%, 15.0 \%, 50.0 \%, 40.9 \%$, and $54.1 \%$, respectively; in stage assessment by $23.0 \%, 25.0 \%, 35.0 \%, 83.3 \%, 54.5 \%$, and $79.1 \%$, respectively; and in parasitic density assessment by $0 \%, 6.2 \%, 10.0 \%, 33.3 \%, 0 \%$, and $12.5 \%$, respectively. We conclude that in the period under evaluation, the percentage of laboratories that diagnosed and recognized the species and stage increases, which is not the case for recognition of parasitic density.
\end{abstract}

Keywords: Malaria; Diagnosis; Laboratories; Peru; Plasmodium (source: MeSH NLM).

\section{INTRODUCCIÓN}

La malaria o paludismo es una enfermedad causada por parásitos del género Plasmodium cuyas cinco especies se transmiten por la picadura de mosquitos del género Anopheles, siendo potencialmente mortal en algunas condiciones y grupos poblacionales. En regiones endémicas, la malaria se debe principalmente a infección por $P$. vivax y $P$. falciparum ${ }^{(1,2)}$. Según el informe mundial sobre el paludismo de la Organización Mundial de la Salud (OMS), en 2016 existieron cerca de 216 millones de casos y 445 mil muertes a causa del paludismo ( $90 \%$ ocurrieron en África) ${ }^{(1,3)}$, siendo un problema de salud pública en África, Asia Sudoriental, Mediterráneo Oriental, Pacífico Occidental y las Américas (4). En Perú, se registraron 55225 casos y diez defunciones, a predominio de infección por $P$. vivax en $2017^{(5)}$.

La Estrategia Técnica Mundial contra la Malaria 20162030 de la OMS, propone la reducción de las tasas de incidencia y mortalidad en un $90 \%$ para el 2030; para

\footnotetext{
Centro Nacional de Salud Pública, Instituto Nacional de Salud. Lima, Perú.

Red Prestacional Sabogal, Seguro Social de Salud EsSalud. Lima, Perú.

Facultad de Medicina, Universidad Nacional Mayor de San Marcos. Lima, Perú.

Licenciado en Biología; ${ }^{b}$ médico cirujano; ${ }^{c}$ licenciada en Biología, Microbiología y Parasitología; ${ }^{\text {d }}$ técnico de laboratorio.

Recibido: 16/10/2018 Aprobado: 28/08/2019 En línea: 23/09/2019
}

Citar como: Bartra-More CA, Ramos-Castillo J, Mendoza-Bautista RC, Galindo-Cabello NR, Gebol-Cahuaza M, Chirinos-Palomino FJ. Evaluación del desempeño en el diagnóstico microscópico de malaria en la red de laboratorios del Instituto Nacional de Salud de Perú, 2012-2017. Rev Peru Med Exp Salud Publica. 2019;36(3):469-74. doi: http://dx.doi.org/10.17843/rpmesp.2019.363.3989. 
ello, fundamenta sus objetivos en la prevención mediante el control vectorial, la quimioprofilaxis y el diagnóstico oportuno. Dentro de las propuestas se recomienda el diagnóstico y tratamiento precoz de los pacientes con malaria ${ }^{(3,6)}$. Asimismo, se establecieron una serie de lineamientos por parte de la OMS, para asegurar la calidad del diagnóstico microscópico de malaria, mediante la evaluación del desempeño ${ }^{(7)}$.

La gota gruesa es método de elección para el diagnóstico microscópico de malaria por su bajo costo, rapidez, determinación de estadios parasitarios y densidad parasitaria; pero su ejecución y efectividad diagnóstica necesita de entrenamiento y las competencias correspondientes que requieren ser evaluados de manera periódica para garantizar la calidad en el diagnóstico de malaria ${ }^{(7,8)}$.

En Perú, el Instituto Nacional de Salud (INS) dirige la red nacional de laboratorios especializados de referencia regional ylaboratorios de referencia nacional de Sudamérica. Es así que el presente estudio tiene como objetivo evaluar el desempeño en el diagnóstico microscópico de malaria de los laboratorios pertenecientes al Laboratorio de Referencia Nacional de Malaria (LRNM) del INS durante los años 20122017, de regiones endémicas y no endémicas de malaria, en el marco del Programa de evaluación externa del desempeño para el diagnóstico microscópico de malaria promovido por la OMS, con la finalidad de contribuir a un mejor análisis del componente diagnóstico como estrategia de reducción de la morbimortalidad por malaria.

\section{EL ESTUDIO}

Se realizó un estudio descriptivo y de concordancia. Durante el periodo 2012-2017, se evaluó el desempeño de los laboratorios adscritos al LRNM del INS, de ubicación en capitales de provincia de nuestro país y descritos en las Tablas 1 y 2 .

Se enviaron paneles de 20 láminas estandarizadas (parásito y parasitemia definida por microscopía y reacción en cadena de polimerasa) elaboradas por el LRNM, a cada uno de los observadores de los laboratorios. Las 20 láminas estuvieron compuestas de: láminas con $P$. falciparum, láminas con $P$. vivax, láminas con ambas especies, y láminas negativas. El envío de los paneles y evaluación a los laboratorios se realizó anualmente. Si bien existen varios métodos de evaluación de desempeño microscópico de malaria, el uso de paneles de láminas se realizó según el manual recomendado por la OMS ${ }^{(7)}$.

Se evaluaron los siguientes cuatro parámetros en el desempeño del diagnóstico microscópico de malaria: diagnóstico microscópico de resultado (positivo o negativo), identificación de especie de Plamodium ( $P$. vivax, $P$.

\section{MENSAJES CLAVE}

Motivación para realizar el estudio. La estrategia de control de malaria requiere el diagnóstico oportuno en los establecimientos de salud. En un país endémico como el nuestro, es importante contar con las capacidades adecuadas para un rápido diagnóstico y tratamiento, acortar el tiempo de transmisión y prevenir la propagación de esta patología.

Principales hallazgos. Entre 2012 y 2017 se incrementó el porcentaje de laboratorios que diagnosticaron y reconocieron la especie y estadio de $P$. vivax y $P$. falciparum, mas no el reconocimiento de la densidad parasitaria.

Implicancias. Sin una adecuada determinación de la densidad parasitaria en malaria, no es posible evaluar la eficacia del tratamiento antiparasitario.

falciparum, o infecciones mixtas), identificación de estadios de Plasmodium (asexuado o sexuado), y determinación de la densidad parasitaria expresada en parásitos por microlitro (considerando una diferencia no mayor de $50 \%$ entre una lectura y otra) ${ }^{(9)}$. La obtención de resultados de los parámetros evaluados fue en tiempo real en cada año descrito, a través del sistema electrónico NetLab del INS de acceso y monitoreo por los autores.

Se determinó el porcentaje de concordancia tomando en consideración el resultado del número de láminas concordantes entre los observadores de los laboratorios y el LRNM, dividido por el total de muestras enviadasymultiplicado por $100 \%$. Se utilizó el coeficiente kappa (k) como medida estadística de grado de concordancia observada entre las variables cualitativas descritas. Si el valor del coeficiente $k$ es cercano a +1 , mayor es el grado de concordancia entre la observación de las láminas enviadas y las observadas; valores $\mathrm{k}$ entre 0,4 y 0,6 indican concordancia moderada; entre 0,61 y 0,8 indican concordancia buena; entre 0,81 y 1 indican concordancia muy buena $(10,11)$.

El análisis estadístico e interpretación de la concordancia se realizó en base a lo recomendado por la OMS (11). Luego de la determinación del porcentaje de concordancia, los laboratorios evaluados fueron calificados como aceptable o no aceptable según lo propuesto por el Programa Regional de Malaria de la Organización Panamericana de la Salud (OPS) (11). Así, para el parámetro de resultado se consideró aceptable una concordancia $>95 \%$, para el parámetro de especie se consideró $>95 \%$, para el parámetro estadio se consideró $>80 \%$, y para la evaluación del parámetro densidad parasitaria se consideró $>80 \%$.

Se mantuvo la confidencialidad de los datos de los observadores de los laboratorios evaluados, los mismos que firmaron un consentimiento informado. Los datos obtenidos forman parte de los informes anuales de dicha evaluación. 
Tabla 1. Porcentajes de concordancia de resultado e identificación de especie en el diagnóstico microscópico de malaria, 2012-2017.

\begin{tabular}{|c|c|c|c|c|c|c|c|c|c|c|c|c|}
\hline \multirow{2}{*}{ Laboratorio } & \multicolumn{6}{|c|}{ Diagnóstico microscópico de resultado (+/-) } & \multicolumn{6}{|c|}{ Identificación de especie* } \\
\hline & 2012 & 2013 & 2014 & 2015 & 2016 & 2017 & 2012 & 2013 & 2014 & 2015 & 2016 & 2017 \\
\hline Alto Amazonas - Yurimaguas & - & 100 & 100 & 100 & 100 & 100 & - & 94,6 & 100 & 100 & 100 & 100 \\
\hline Cusco & - & 95,0 & 95,0 & 95,0 & 100 & 100 & - & 71,4 & 70,8 & 73,3 & 82,1 & 100 \\
\hline Cusco - La Convención & - & 90,0 & 100 & 100 & 100 & 100 & - & 67,9 & 91,7 & 92,9 & 96,4 & 100 \\
\hline Loreto - Iquitos & - & 100 & 100 & 90,0 & 100 & 100 & - & 82,1 & 96,4 & 92,9 & 96,4 & 100 \\
\hline Tumbes & 90,0 & - & 100 & 100 & 100 & 100 & 64,3 & - & 100 & 96,4 & 100 & 100 \\
\hline Amazonas - Chachapoyas & 85,0 & - & 80,0 & - & 100 & 100 & 57,1 & - & 57,1 & - & 89,3 & 78,5 \\
\hline Ayacucho & 95,0 & - & 80,0 & - & 80,0 & 100 & 60,7 & - & 50,0 & - & 82,1 & 53,5 \\
\hline Cajamarca & 85,0 & - & - & 100 & 100 & 100 & 71,4 & - & & 100 & 96,4 & 89,2 \\
\hline Cajamarca - Jaén & 90,0 & - & 85,0 & - & 95,0 & 100 & 78,6 & - & 82,1 & - & 71,4 & 85,7 \\
\hline Huánuco & - & 90,0 & 90,0 & - & 100 & 100 & - & 78,6 & 42,9 & - & 89,3 & 89,2 \\
\hline Junín - Satipo & - & 90,0 & 90,0 & - & 100 & 100 & - & 82,1 & 67,9 & - & 85,7 & 100 \\
\hline Lambayeque & - & 95,0 & 95,0 & - & 100 & 95,0 & - & 89,3 & 78,6 & - & 96,4 & 89,2 \\
\hline Madre de Dios & 100 & - & 95,0 & - & 100 & 100 & 75,0 & - & 78,6 & - & 100 & 96,4 \\
\hline Piura LARESA & 75,0 & - & 85,0 & - & 95,0 & 100 & 60,7 & - & 75,0 & - & 75,0 & 82,1 \\
\hline Piura - Sullana & 95,0 & - & $100 \%$ & - & 95,0 & 100 & 89,3 & - & 92,9 & - & 82,1 & 89,2 \\
\hline San Martín & 100 & - & 100 & - & 95,0 & 100 & 92,9 & - & 92,9 & - & 96,4 & 96,4 \\
\hline Ucayali & - & 95,0 & 100 & - & 60,0 & 80 & - & 82,1 & 78,6 & - & 60,7 & 85,7 \\
\hline Ancash & - & 80,0 & - & - & 95,0 & 95 & - & 53,6 & - & - & 60,7 & 71,4 \\
\hline Apurímac - Abancay & - & - & 95,0 & - & 100,0 & 100 & - & - & 71,4 & - & 64,3 & 100 \\
\hline Apurímac - Andahuaylas & - & - & 90,0 & - & 100 & 100 & - & - & 57,1 & - & 78,6 & 100 \\
\hline Amazonas - Bagua & 90,0 & - & 90,0 & - & - & 100 & 67,9 & - & 85,7 & - & - & 100 \\
\hline La Libertad & 85,0 & - & 95,0 & - & - & 100 & 78,6 & - & 71,4 & - & - & 92,8 \\
\hline Pasco - Oxapampa & - & 100 & - & - & 100 & 100 & - & 96,4 & - & - & 78,6 & 100 \\
\hline Arequipa & - & 60,0 & - & - & - & - & - & 39,3 & - & - & - & - \\
\hline Huancavelica & - & 100 & - & - & - & - & - & 75,0 & - & - & - & - \\
\hline Ica & - & 70,0 & - & - & - & - & - & 35,7 & - & - & - & - \\
\hline Junín & - & - & - & - & 100 & 100 & - & - & - & - & 100 & 100 \\
\hline Lima Ciudad & 80,0 & - & - & - & - & - & 57,1 & - & - & - & - & - \\
\hline Lima Este & 95,0 & - & - & - & - & - & 71,4 & - & - & - & - & - \\
\hline Moquegua & - & 90,0 & - & - & - & - & - & 60,7 & - & - & - & - \\
\hline Puno & - & 65,0 & - & - & - & - & - & 53,6 & - & - & - & - \\
\hline Tacna & - & 75,0 & - & - & - & - & - & 17,9 & - & - & - & - \\
\hline
\end{tabular}

Los valores presentados en la tabla corresponden a porcentajes.

* $P$. vivax, P. falciparum, o infecciones mixtas.

Concordancia de diagnóstico microscópico de resultado aceptable de $95 \%$ a $100 \%$. Concordancia de identificación de especie aceptable de $95 \%$ a $100 \%$. Coeficiente de kappa $\geq 0,6$ en el análisis de concordancia de diagnóstico microscópico de resultado y de identificación de especie.

Guión: dato no disponible

\section{HALLAZGOS}

Entre los años 2012 a 2017 participaron un total de 13, $16,20,6,22$, y 24 laboratorios, respectivamente. El porcentaje de concordancia hallado para el diagnóstico microscópico de Plasmodium para dicho periodo fue de $38,4 \%, 43,7 \%, 60,0 \%, 83,3 \%, 90,9 \%$, y $95,8 \%$ de los laboratorios, respectivamente, y tuvieron la calificación de aceptable. Asimismo, para la evaluación de la especie de Plasmodium, tuvieron la calificación de aceptable el $0 \%, 6,2 \%, 15 \%, 50 \%, 40,9 \%$, y $54,1 \%$ de los laboratorios, según se muestra en la Tabla 1.
En relación a una calificación aceptable de la evaluación del desempeño en el diagnóstico microscópico del estadio de Plasmodium, se encontró en el 23\%, 25\%, 35\%, 83,3\%, $54,5 \%$, y $79,1 \%$ de los laboratorios, respectivamente. Asimismo, una evaluación aceptable de la densidad parasitaria de Plasmodium se encontró en el $0 \%$, $6,2 \%, 10 \%, 33,3 \%, 0 \%$, y $12,5 \%$ de los laboratorios, respectivamente, tal como se observa en la Tabla 2.

Para la evaluación de los parámetros resultado, especie y estadio, se halló un coeficiente $\mathrm{k} \geq 0,6$ en el análisis de concordancia. Para el parámetro densidad parasitaria, no se evaluó el grado de concordancia debido al bajo porcentaje de concordancia. 
Tabla 2. Porcentajes de concordancia de estadio y densidad parasitaria en el diagnóstico microscópico de malaria, 2012-2017.

\begin{tabular}{|c|c|c|c|c|c|c|c|c|c|c|c|c|}
\hline \multirow[t]{2}{*}{ Laboratorio } & \multicolumn{6}{|c|}{$\begin{array}{l}\text { Identificación de estadio } \\
\text { (sexuado o asexuado) }\end{array}$} & \multicolumn{6}{|c|}{ Densidad parasitaria (parásitos $/ \mu \mathrm{L}$ ) } \\
\hline & 2012 & 2013 & 2014 & 2015 & 2016 & 2017 & 2012 & 2013 & 2014 & 2015 & 2016 & 2017 \\
\hline Alto Amazonas -Yurimaguas & - & 82,1 & 89,6 & 96,4 & 89,3 & 94,6 & - & 62,5 & 81,3 & 80,4 & 69,6 & 69,6 \\
\hline Cusco & - & 71,4 & 68,8 & 76,7 & 80,4 & 94,6 & - & 51,8 & 37,5 & 48,3 & 53,6 & 83,9 \\
\hline Cusco - La Convención & - & 69,6 & 83,3 & 87,5 & 92,9 & 87,5 & - & 55,4 & 70,8 & 55,4 & 50,0 & 67,8 \\
\hline Loreto - Iquitos & - & 69,6 & 89,3 & 87,5 & 85,7 & 91,0 & - & 50 & 82,1 & 71,4 & 75,0 & 71,4 \\
\hline Tumbes & 78,6 & - & 91,1 & 94,6 & 87,5 & 98,2 & 41,1 & - & 71,4 & 57,1 & 60,7 & 83,9 \\
\hline Amazonas - Chachapoyas & 62,5 & - & 64,3 & - & 73,2 & 67,8 & 26,8 & - & 41,1 & - & 58,9 & 37,5 \\
\hline Ayacucho & 66,1 & - & 60,7 & - & 73,2 & 64,2 & 28,6 & - & 26,8 & - & 30,4 & 19,6 \\
\hline Cajamarca & 69,6 & - & - & 96,7 & 82,1 & 83,9 & 42,9 & - & & 85,0 & 57,1 & 64,2 \\
\hline Cajamarca - Jaén & 78,6 & - & 78,6 & - & 62,5 & 78,5 & 55,4 & - & 53,6 & - & 46,4 & 53,5 \\
\hline Huánuco & - & 71,4 & 58,9 & - & 66,1 & 82,1 & - & 51,8 & 21,4 & - & 53,6 & 64,2 \\
\hline Junín - Satipo & - & 80,4 & 76,8 & - & 85,7 & 89,2 & - & 60,7 & 44,6 & - & 53,6 & 67,8 \\
\hline Lambayeque & - & 87,5 & 71,4 & - & 83,9 & 82,1 & - & 62,5 & 42,9 & - & 42,9 & 48,2 \\
\hline Madre de Dios & $73,2 \%$ & - & 71,4 & - & 87,5 & 94,6 & 32,1 & - & 53,6 & - & 67,9 & 69,6 \\
\hline Piura LARESA & 71,4 & - & 75,0 & - & 67,9 & 82,1 & 37,5 & - & 55,4 & - & 23,2 & 55,3 \\
\hline Piura - Sullana & 85,7 & - & 87,5 & - & 83,9 & 83,9 & 62,5 & - & 69,6 & - & 42,9 & 55,3 \\
\hline San Martín & 80,4 & - & 80,4 & - & 83,9 & 87,5 & 69,6 & - & 69,6 & - & 60,7 & 67,8 \\
\hline Ucayali & - & 71,4 & 73,2 & - & 57,1 & 78,5 & - & 55,4 & 46,4 & - & 32,1 & 60,7 \\
\hline Ancash & - & 67,9 & - & - & 55,4 & 62,5 & - & 25,0 & & - & 35,7 & 37,5 \\
\hline Apurímac - Abancay & - & - & 82,1 & - & 67,9 & 85,7 & - & - & 62,5 & - & 44,6 & 60,7 \\
\hline Apurímac - Andahuaylas & - & - & 67,9 & - & 75,0 & 82,1 & - & - & 37,5 & - & 48,2 & 64,2 \\
\hline Amazonas - Bagua & 69,6 & - & 75,0 & - & - & 85,7 & 48,2 & - & 51,8 & - & - & 78,5 \\
\hline La Libertad & 83,9 & - & 78,6 & - & - & 87,5 & 51,8 & - & 57,1 & - & - & 48,2 \\
\hline Pasco - Oxapampa & - & 96,4 & - & - & 71,4 & 91,0 & - & 85,7 & - & - & 28,6 & 67,8 \\
\hline Arequipa & - & 64,3 & - & - & - & - & - & 25 & - & - & - & - \\
\hline Huancavelica & - & 75,0 & - & - & - & - & - & 44,6 & - & - & - & - \\
\hline Ica & - & 57,1 & - & - & - & - & - & 17,9 & - & - & - & - \\
\hline Junín & - & - & - & - & 94,6 & 96,4 & - & - & - & - & 75,0 & 83,9 \\
\hline Lima Ciudad & 67,9 & - & - & - & - & - & 35,7 & - & - & - & - & - \\
\hline Lima Este & 73,2 & - & - & - & - & - & 46,4 & - & - & - & - & - \\
\hline Moquegua & - & 57,1 & - & - & - & - & - & 39,3 & - & - & - & - \\
\hline Puno & - & 66,1 & - & - & - & - & - & 28,6 & - & - & - & - \\
\hline Tacna & - & 41,1 & - & - & - & - & - & 5,4 & - & - & - & - \\
\hline
\end{tabular}

Los valores presentados en la tabla corresponden a porcentajes.

Concordancia de estadio aceptable de $80 \%$ a $100 \%$. Concordancia de densidad parasitaria aceptable de $80 \%$ a $100 \%$.

Coeficiente de kappa $\geq 0,6$ en el análisis de concordancia de estadio. No se evaluó el grado de concordancia para la densidad parasitaria.

Guión: dato no disponible

\section{DISCUSIÓN}

Según la OMS, el primer componente de la estrategia global del control de malaria es el acceso al diagnóstico y tratamiento oportuno en todos los establecimientos de salud ${ }^{(6,11)}$. En un país endémico como el nuestro, es importante contar con las capacidades adecuadas para un rápido diagnóstico y tratamiento, así como para acortar el tiempo de transmisión y prevenir la propagación.

El examen microscópico de malaria es la prueba patrón de oro que continúa siendo útil para el diagnóstico de la enfermedad, al ser sencilla de ejecutar y tener una alta sensibilidad y especificidad, es accesible para ser ejecutada por todo el personal de salud desde un establecimiento básico a uno de mayor complejidad (7). En el Perú, la Norma Técnica de Salud № 082-2010 del Ministerio de Salud, brinda pautas para el control de calidad y diagnóstico microscópico de malaria ${ }^{(12)}$.

Los resultados del presente estudio muestran un incremento del número de laboratorios que durante el periodo 20122017 cumplieron con la calificación de aceptable para el diagnóstico microscópico, reconocimiento de especie y reconocimiento del estadio, mas no en lo referido al reconocimiento de la densidad parasitaria. En relación al reconocimiento de especie, similares resultados se obtuvieron en un estudio de evaluación de desempeño de microscopistas de malaria en laboratorios públicos de referencia en Colombia ${ }^{(8)}$, con la determinación de 
parásitos al 96,9\% e identificación de especies al 88,7\%. Sin embargo, en un estudio realizado en la Amazonía peruana, sólo uno de cada tres microscopistas fue competente en el reconocimiento de especie ${ }^{(13)}$. De igual manera, en un estudio realizado en Cuba, se obtuvo porcentajes de concordancia por debajo de $65 \%$ para la identificación de especies de Plasmodium ${ }^{(14)}$. Asimismo, en un estudio realizado en Etiopía, la concordancia entre el laboratorio de referencia y los profesionales de laboratorio alcanzó sólo el $79 \%{ }^{(15)}$. Los laboratorios evaluados en nuestro estudio mostraron concordancias mayores a $95 \%$ tanto en el reconocimiento de Plasmodium como en la identificación de especie y estadio, existiendo un incremento de porcentaje de aceptabilidad de laboratorios; estos hallazgos ocurren incluso en zonas de menor prevalencia de esta enfermedad. Esta mejoría en la evaluación del desempeño del diagnóstico microscópico se puede atribuir a factores como menor rotación de personal, capacitación y asesoría permanente por el LRNM, así como por la constante evaluación.

Una correcta evaluación de la densidad parasitaria es útil para determinar la severidad de la infección malárica y evaluar la eficacia del tratamiento antiparasitario. Si el tratamiento es eficaz, la densidad parasitaria disminuirá de manera progresiva. Asimismo, una adecuada evaluación de la densidad parasitaria es importante en la infección por $P$. falciparum la cual se asocia con una enfermedad potencialmente mortal, por lo que resulta útil su medición en el proceso de tratamiento. Existen dos métodos para la determinación de la densidad parasitaria: método simple en cruces (semicuantitativo) y el cálculo de parásitos por microlitro (mayor precisión) (12). Nuestros resultados reportan un bajo porcentaje de laboratorios con calificación aceptable en la evaluación del desempeño diagnóstico de este parámetro; es decir, existe aún dificultad técnica de medición mediante parásitos por microlitro, probablemente debido a que en los últimos años se ha normado el segundo método como estándar de medición, presentándose dificultades para dejar de utilizar el método tradicional semicuantitativo. Se requiere de entrenamiento adicional para el logro de tal competencia, la misma que sería efectiva mediante asesoría individualizada así como la evaluación permanente, que no se realiza en la actualidad.

Dentro de las limitaciones del estudio, existieron laboratorios (a predominio de zonas no endémicas) que se evaluaron inicialmente pero que en años posteriores dejaron de ser evaluados por carencia y rotación de personal, falta de respuesta a la evaluación, y dificultades en la comunicación de resultados. En dichas áreas no endémicas, el desempeño del diagnóstico de malaria fue bajo, recomendándose para estas áreas el uso de las pruebas rápidas para el diagnóstico de Plasmodium ${ }^{(16,17)}$, con la finalidad de diagnosticar adecuadamente esta enfermedad hasta que el personal de laboratorio logre las competencias deseadas. Asimismo, la rotación de personal podría determinar una inadecuada evaluación en el periodo de estudio, el $79 \%$ de observadores se mantuvieron en el estudio, aunque es preciso mencionar que desde el 2015 el Ministerio de Salud tiene como estrategia establecer personal permanente en los laboratorios regionales del Perú según lo recomendado por OMS/OPS.

Se concluye que durante el periodo 2012-2017, los laboratorios evaluados mejoraron el desempeño en el diagnóstico y reconocimiento de especie y estadio de $P$. vivax y $P$. falciparum. Sin embargo, existe un bajo porcentaje de laboratorios con calificación aceptable en la evaluación de la densidad parasitaria. Tal como han reportado algunas investigaciones, el entrenamiento y la capacitación constituyen alternativas para mejorar el desempeño microscópico en el diagnóstico de la malaria ${ }^{(18)}$.

Contribuciones de autoría: CBM y FCP participaron en la concepción del estudio. JRC, RMB, NGC, FCP y MGC participaron en la interpretación de los datos, JRC participó en la redacción del artículo y todos los autores participaron en la aprobación final del artículo redactado.

Fuentes de financiamiento: Instituto Nacional de Salud.

Conflictos de interés: los autores declaran no tener conflictos de interés.

\section{REFERENCIAS BIBLIOGRÁFICAS}

1. Baird JK, Valecha N, Durpac S, White NJ, Price RN. Diagnosis and Treatment of P. vivax Malaria. Am J Trop Med Hyg. 2016;95(Suppl 6):35-51. doi: https:// doi.org/10.4269/ajtmh.16-0171

2. Organización Mundial de la Salud OMS [Internet]. Ginebra: Organización Mundial de la Salud; 2017 [citado el 27 de julio de 2019]. Disponible en: http:// www.who.int/mediacentre/factsheets/ fs094/es/

3. Organización Mundial de la Salud. Paludismo. Puntos clave: Informe mundial sobre el paludismo 2017 [Internet]. Ginebra: Organización Mundial de la Salud; 2017 [citado el 27 de julio de 2019]. Disponible en: http://www.who.int/malaria/media/ world-malaria-report-2017/es/

4. Recht J, Siqueira AM, Monteiro W, Herrera SM, Herrera S, Lacerda M. Malaria in Brazil, Colombia, Peru and Venezuela: current challenges in malaria control and elimination. Malar J. 2017;16(1):273. doi: https://doi. org/10.1186/s12936-017-1925-6.
5. Centro Nacional de Epidemiología, Prevención y Control de Enfermedades. Enfermedades metaxénicas: Malaria [Internet]. Lima: Centro Nacional de Epidemiología, Prevención y Control de Enfermedades, Ministerio de Salud del Perú; 2018 [citado el 24 de julio de 2018]. Disponible en: http://www.dge.gob.pe/portal/index. php?option $=$ com_content $\&$ view $=$ article\&id $=14 \&$ Itemid $=154$.

6. Organización Mundial de la Salud. Estrategia técnica mundial contra 
la malaria 2016-2030 [Internet]. Ginebra: Organización Mundial de la Salud; 2015 [citado el 24 de julio de 2018]. Disponible en: http:// apps.who.int/iris/bitstream/han$\mathrm{dle} / 10665 / 186671 / 9789243564999$ spa.pdf; jessionid=1F61D35186FADA3EB02EBAC002545C23?sequence $=1$.

7. World Health Organization. Malaria microscopy quality assurance manual - Version 2 [Internet]. Ginebra: World Health Organization; 2016 [citado el 24 de julio de 2018]. Disponible en: http://apps.who.int/iris/bitstream/handle/10665/204266/9789241549394_ eng.pdf ?sequence $=1$.

8. Mendoza NM, Gonzáles NE. Evaluación de desempeño mediante paneles de láminas: una herramienta para la clasificación de los microscopistas senior del Programa de Control de la Malaria en Colombia. Biomédica. 2015;35(4):5829. doi: https://dx.doi.org/10.7705/ biomedica.v35i4.2694

9. Organización Panamericana de la Salud. Guía práctica revisada para estudios de eficacia de los medicamentos antimaláricos en las Américas [Internet]. Organización Panamericana de la Salud; 2010 [citado el 23 de agosto de 2018]. Disponible en: http://www1.paho.org/ $\mathrm{hq} /$ dmdocuments/2011/modificadaPAN-JUL-2010-integrada-2011.pdf.

10. Landis JR, Koch GG. The measurement of observer agreement for categorical data. Biometrics. 1977;33(1):159-174.
11. Programa Regional de Malaria Prevención y Control de Enfermedades Transmisibles, Vigilancia de la Salud, y Prevención y Control de Enfermedades de la Organización Panamericana de la Salud Organización Panamericana de la Salud. Programa de Evaluación Externa del Desempeño para el Diagnóstico Microscópico de Malaria [Internet]. Organización Panamericana de la Salud; 2010 [citado el 23 de agosto de 2018]. Disponible en: https://www.paho. org/hq/dmdocuments/2013/PEEDMalaria-OPS-Esp-2011-1.pdf.

12. Instituto Nacional de Salud. Norma Técnica de Salud No 082-MINSA/ INS-V.01. Norma Técnica de Salud para el control de calidad del diagnóstico microscópico de malaria [Internet]. Lima: Ministerio de Salud del Perú; 2010 [citado el 27 de julio de 2019]. Disponible en: http://www. ins.gob.pe/insvirtual/images/otrpubs/ pdf/FINAL \%20MALARIA \%20 28.12.10\%5B1\%5D.pdf

13. Rosas-Aguirre A, Gamboa D, Rodríguez H, Llanos-Zavalaga $\mathrm{F}$, Aguirre K, Llanos-Cuentas A. Uso de paneles de láminas estandarizadas para la evaluación de competencias en el diagnóstico microscópico de malaria en la amazonía peruana. Rev Peru Med Exp Salud Pública. 2010;27(4): 540-47.

14. Rojas L, Ginorio D, Nuñez F, Valdespino M, Hernández N, Sánchez M, Montoto V. Evaluación de las habilidades prácticas para realizar el diagnóstico microscópico de malaria en tres provincias de la
República de Cuba. Rev Cubana Med Trop. 2017;69(3):1-13.

15. Alemu M, Tadesse D, Hailu T, Mulu W, Derbie A, Hailu T, Abera B. Performance of laboratory professionals working on malaria microscopy in Tigray, North Ethiopia. J Parasitol Res. 2017;2017:9064917. doi: 10.1155/2017/9064917.

16. Gonzáles-Cerón L, Rodríguez M, Betanzos A, Abadía A. Eficacia de una prueba rápida para el diagnóstico de P. vivax en pacientes sintomáticos de Chiapas, México. Salud Publica Mex. 2005;47(4):282-87

17. Mendoza N, Rosas A, Burgos J. Evaluación de la aplicación de pruebas de diagnóstico rápido para malaria como parte de una estrategia integral para su control en Colombia. Biomédica. 2011;31:55-69. doi: https://doi.org/10.7705/biomedica. v31i1.336

18. Nazaré G, da Silva F, Ferreira C, Rafael P, Fortes F, Rojas L. Evaluación de la calidad del diagnóstico de la malaria por microscopía óptica en laboratorios provinciales de la República de Angola. Rev Cubana Med Trop. 2014;66(2):191-201

Correspondencia: Carlos Bartra More Dirección: Aurelio Miroquezada 539 Urb. Ingenieria.San Martín de Porres. Lima, Perú.

Teléfono: 5117481111 Anexo 2168

Correo electrónico: carlosarturo777@gmail.com 